\title{
ON MAPPING LINEAR PARTIAL DIFFERENTIAL EQUATIONS TO CONSTANT COEFFICIENT EQUATIONS*
}

\author{
GEORGE W. BLUMAN $\dagger$
}

\begin{abstract}
A constructive algorithm is developed to determine whether or not a given linear p.d.e. can be mapped into a linear p.d.e. with constant coefficients. The algorithm is based on analyzing the infinitesimals of the Lie group of transformations leaving invariant the given p.d.e. As consequences, in two dimensions, necessary and sufficient conditions are given for mapping:

(1) a parabolic p.d.e. into the heat equation;

(2) a hyperbolic p.d.e. into the wave equation;

(3) an elliptic p.d.e. into Laplace's equation or the Helmholtz equation.

The corresponding mappings are given explicitly.
\end{abstract}

1. Introduction. In this paper we develop a constructive algorithm for answering the question of whether or not a given linear partial differential equation can be mapped into a partial differential equation (p.d.e.) with constant coefficients.

A constant coefficient p.d.e. with $n$ independent variables is invariant under the $n$-parameter Lie group of translations of its independent variables. Hence it is necessary that a given p.d.e. with $n$ independent variables be invariant under at least an $n$-parameter Lie group of point transformations in order to have a mapping to a constant coefficient p.d.e. The constructive algorithm presented in this paper is based on the idea of mapping infinitesimals of the Lie group of point transformations leaving invariant a given linear p.d.e. into infinitesimals corresponding to invariance under translations.

For examples we consider the parabolic, hyperbolic, and elliptic equations in two dimensions. In the respective cases necessary and sufficient conditions are given for the variable coefficients so that a mapping to a constant coefficient p.d.e. is possible. In addition each corresponding mapping is given explicitly.

2. The mapping algorithm. Consider an $m$ th order homogeneous linear p.d.e. with $n$ independent variables $x=\left(x_{1}, x_{2}, \cdots, x_{n}\right)$ and dependent variable $u(x)$ :

$$
A(x) u+A^{i_{1}}(x) u_{i_{1}}+\cdots+A^{i_{1} i_{2} \cdots i_{m}}(x) u_{i_{1} i_{2} \cdots i_{m}}=0,
$$

where

$$
\begin{gathered}
A(x), \quad A^{i_{1} i_{2} \cdots i_{k}}(x): \quad \mathbb{R}^{n} \rightarrow \mathbb{R}, \\
u_{i_{1} i_{2} \cdots i_{k}}=\frac{\partial^{k} u}{\partial x_{i_{1}} \partial x_{i_{2}} \cdots \partial x_{i_{k}}}, \quad i_{k}=1,2, \cdots, n,
\end{gathered}
$$

$k=1,2, \cdots, m, x \in$ domain $D \subset \mathbb{R}^{n}$. (Throughout this paper repeated indices are summed from 1 to $n$.)

Our aim is to map (1) (and determine whether or not this is possible) into a constant coefficient linear p.d.e. with respect to some new independent variables $\xi=\left(\xi_{1}, \xi_{2}, \cdots, \xi_{n}\right)$ and new dependent variable $U(\xi)$ :

$$
B U+B^{i_{1}} U_{i_{1}}+\cdots+B^{i_{1} i_{2} \cdots i_{m}} U_{i_{1} i_{2} \cdots i_{m}}=0,
$$

* Received by the editors September 24, 1982. This research was supported in part by the Research Council of the United Kingdom and the Natural Sciences and Engineering Research Council of Canada.

† Department of Theoretical Mechanics, University of Nottingham, Nottingham, United Kingdom NG7 2RD. Permanent address: Department of Mathematics, University of British Columbia, Vancouver, British Columbia, Canada V6T 1Y4. 
where $\left\{B, B^{i_{1} i_{2} \cdots i_{k}}\right\}$ are all constants,

$$
U_{i_{1} i_{2} \cdots i_{k}}=\frac{\partial^{k} U}{\partial \xi_{i_{1}} \partial \xi_{i_{2}} \cdots \partial \xi_{i_{k}}}, \quad i_{k}=1,2, \cdots, n, \quad k=1,2, \cdots, m .
$$

The mapping is given by

$$
x=x(\xi), \quad u(x)=F(\xi) U(\xi)
$$

where $u(x)$ solves $(1)$ in domain $D$ if and only if $U(\xi)$ solves (2) in domain $\mathscr{D}$. The one-to-one mapping $x(\xi)$ transforms domain $\mathscr{D}$ into domain $D$ with inverse mapping given by $\xi(x) . F(\xi)$ is called the multiplier of the mapping (3).

The constant coefficient p.d.e. (2) has the property of being invariant under the $n$-parameter Lie group of translations

$$
\xi_{i}^{*}=\xi_{i}+\varepsilon_{j} \delta_{i j}, \quad U^{*}=U,
$$

$i=1,2, \cdots, n$, where $\left\{\varepsilon_{1}, \varepsilon_{2}, \cdots, \varepsilon_{n}\right\}$ are the $n$ parameters. Hence it is necessary that (1) be invariant under at least an $n$-parameter Lie group of point transformations in order to have a mapping into some constant coefficient p.d.e. (2). Moreover, under the mapping (3) (to be determined) some representation of the Lie group leaving (1) invariant must be transformed into the $n$-parameter Lie group of translations (4). In particular, for some representation, the infinitesimals of the Lie group leaving (1) invariant must be transformed into the corresponding infinitesimals for translation invariance.

Given a linear p.d.e. (1), there is an algorithm to find its invariance Lie group of point transformations. This group is of the form

$$
\begin{aligned}
& x_{i}^{*}=x_{i}+\varepsilon X_{i}(x)+O\left(\varepsilon^{2}\right), \quad i=1,2, \cdots, n \\
& u^{*}=u+\varepsilon g(x) u+O\left(\varepsilon^{2}\right)
\end{aligned}
$$

where the infinitesimals $\left\{X_{i}(x), g(x)\right\}$ correspond to the solution of a set of determining equations. (Bluman and Cole (1974), Ovsjannikov (1962)). If p.d.e. (1) is invariant under at least an $n$-parameter Lie group of point transformations then it is possible to have an $n$-parameter $\left\{\varepsilon_{1}, \varepsilon_{2}, \cdots, \varepsilon_{n}\right\}$ representation of (5) of the form

$$
\begin{aligned}
& \varepsilon X_{i}(x)=\varepsilon_{j} X_{j i}(x), \quad i=1,2, \cdots, n, \\
& \varepsilon g(x)=\varepsilon_{j} g_{j}(x)
\end{aligned}
$$

where $\operatorname{det}\left|X_{i j}(x)\right| \neq 0$ in $D$.

If there is some mapping (3) of p.d.e. (1) into a constant coefficient p.d.e. (2) then it must map a representation (6) of the infinitesimals of the Lie group leaving invariant p.d.e. (1) into the translation group (4). If such a mapping (3) exists then it follows that

$$
\begin{aligned}
& x_{i}^{*}=x_{i}+\varepsilon_{j} X_{j i}(x)+\cdots=x_{i}\left(\xi^{*}\right)=x_{i}+\varepsilon_{j} \delta_{k j} \frac{\partial x_{i}}{\partial \xi_{k}}(\xi)+\cdots, \quad i=1,2, \cdots, n, \\
& u^{*}=u(x)+\varepsilon_{j} g_{j}(x) u(x)+\cdots=F\left(\xi^{*}\right) U(\xi)=u(x)+\varepsilon_{j} \delta_{k j} \frac{\partial F(\xi)}{\partial \xi_{k}} U(\xi)+\cdots
\end{aligned}
$$

Taking each independent parameter $\left\{\varepsilon_{1}, \varepsilon_{2}, \cdots, \varepsilon_{n}\right\}$ in turn, one finds that the mapping 
(3) is given by the set of equations

$$
\frac{\partial x_{i}}{\partial \xi_{j}}=X_{j i}(x), \quad \frac{1}{F} \frac{\partial F}{\partial \xi_{j}}=g_{j}(x), \quad i, j=1,2, \cdots, n .
$$

Thus the necessary and sufficient condition for mapping p.d.e. (1) into a constant coefficient p.d.e. (2) is the existence of a solution $\{x(\xi), F(\xi)\}$ of the system of equations (7). Of more interest is $\xi(x)$ related to $x(\xi)$ by the Jacobian conditions:

$$
\frac{\partial x_{j}}{\partial \xi_{k}} \frac{\partial \xi_{i}}{\partial x_{j}}=\delta_{i k}, \quad i, k=1,2, \cdots, n .
$$

From (7), (8) $\xi(x)$ is found by solving the system of equations

$$
X_{k j}(x) \frac{\partial \xi_{i}}{\partial x_{j}}=\delta_{i k}, \quad i, k=1,2, \cdots, n .
$$

The system (7), and hence (9), has a solution if and only if

$$
\begin{aligned}
& \frac{\partial^{2} x_{i}}{\partial \xi_{j} \partial \xi_{k}}=\frac{\partial^{2} x_{i}}{\partial \xi_{k} \partial \xi_{j}} \\
& \frac{\partial}{\partial \xi_{k}}\left(\frac{1}{F} \frac{\partial F}{\partial \xi_{j}}\right)=\frac{\partial}{\partial \xi_{j}}\left(\frac{1}{F} \frac{\partial F}{\partial \xi_{k}}\right), \quad i, j, k=1,2, \cdots, n .
\end{aligned}
$$

The consistency relations (10), applied to (7), correspond to the infinitesimals $\left\{X_{j i}(x), g_{i}(x)\right\}, \operatorname{det}\left|X_{i j}(x)\right| \neq 0$, satisfying the system of equations

$$
X_{k l} \frac{\partial X_{j i}}{\partial x_{l}}=X_{j l} \frac{\partial X_{k i}}{\partial x_{l}}, \quad X_{k l} \frac{\partial g_{j}}{\partial x_{l}}=X_{j l} \frac{\partial g_{k}}{\partial x_{l}}, \quad i, j, k=1,2, \cdots, n .
$$

The mapping algorithm can be summarized as follows:

(I) Find the determining equations for the infinitesimals of the Lie group of point transformations leaving invariant the given equation (1). (It is unnecessary to solve the determining equations explicitly as will be seen in the examples.)

(II) Find the coefficients of (1) so that the system of equations (11) has a nontrivial solution where det $\left|X_{i j}(x)\right| \neq 0$ in some domain $D$.

(III) Solve the system of equations (9) to find $\xi(x)$.

(IV) Find the multiplier $F(\xi)$ by solving the second set of equations in (7) or equivalently by solving the set of equations

$$
\frac{1}{F} \frac{\partial F}{\partial x_{i}}=g_{j}(x) \frac{\partial \xi_{j}}{\partial x_{i}}, \quad i=1,2, \cdots, n .
$$

(a) The case $n=2$. For the case of two independent variables, $n=2$, we introduce the notation $x_{1} \rightarrow x, x_{2} \rightarrow y, X_{1} \rightarrow X, X_{2} \rightarrow Y, X_{11} \rightarrow X_{1}, X_{21} \rightarrow X_{2}, X_{12} \rightarrow Y_{1}, X_{22} \rightarrow Y_{2}$, $\xi_{1} \rightarrow \xi, \xi_{2} \rightarrow \eta$.

In this notation the mapping equations (7) become

$$
\begin{aligned}
& \frac{\partial x}{\partial \xi}=X_{1}(x, y), \\
& \frac{\partial y}{\partial \xi}=Y_{1}(x, y),
\end{aligned}
$$




$$
\begin{aligned}
& \frac{\partial x}{\partial \eta}=X_{2}(x, y) \\
& \frac{\partial y}{\partial \eta}=Y_{2}(x, y) \\
& \frac{1}{F} \frac{\partial F}{\partial \xi}=g_{1}(x, y) \\
& \frac{1}{F} \frac{\partial F}{\partial \eta}=g_{2}(x, y)
\end{aligned}
$$

where

$$
\operatorname{det}\left|\begin{array}{cc}
X_{1} & X_{2} \\
Y_{1} & Y_{2}
\end{array}\right| \neq 0
$$

in domain $D$.

If a mapping to a constant coefficient p.d.e. is possible, the new independent variables $(\xi(x, y), \eta(x, y))$ correspond to a solution of the system of p.d.e's

$$
\begin{aligned}
& X_{1}(x, y) \frac{\partial \xi}{\partial x}+Y_{1}(x, y) \frac{\partial \xi}{\partial y}=1 \\
& X_{2}(x, y) \frac{\partial \xi}{\partial x}+Y_{2}(x, y) \frac{\partial \xi}{\partial y}=0 \\
& X_{2}(x, y) \frac{\partial \eta}{\partial x}+Y_{2}(x, y) \frac{\partial \eta}{\partial y}=1 \\
& X_{1}(x, y) \frac{\partial \eta}{\partial x}+Y_{1}(x, y) \frac{\partial \eta}{\partial y}=0
\end{aligned}
$$

For some representation of the infinitesimals leaving invariant the corresponding p.d.e. (1), the consistency relations (11) become

$$
\begin{aligned}
& X_{2} \frac{\partial X_{1}}{\partial x}+Y_{2} \frac{\partial X_{1}}{\partial y}=X_{1} \frac{\partial X_{2}}{\partial x}+Y_{1} \frac{\partial X_{2}}{\partial y} \\
& Y_{1} \frac{\partial Y_{2}}{\partial y}+X_{1} \frac{\partial Y_{2}}{\partial x}=Y_{2} \frac{\partial Y_{1}}{\partial y}+X_{2} \frac{\partial Y_{1}}{\partial x} \\
& X_{2} \frac{\partial g_{1}}{\partial x}+Y_{2} \frac{\partial g_{1}}{\partial y}=X_{1} \frac{\partial g_{2}}{\partial x}+Y_{1} \frac{\partial g_{2}}{\partial y}
\end{aligned}
$$

In the following sections of this paper we find the most general second order linear p.d.e. of the form

$$
\begin{aligned}
& a(x, y) \frac{\partial^{2} u}{\partial x^{2}}+2 b(x, y) \frac{\partial^{2} u}{\partial x \partial y}+c(x, y) \frac{\partial^{2} u}{\partial y^{2}} \\
& +d(x, y) \frac{\partial u}{\partial x}+e(x, y) \frac{\partial u}{\partial y}+f(x, y) u=0
\end{aligned}
$$

which can be mapped into a constant coefficient p.d.e. 
According to the sign of $b^{2}-a c$, we can reduce (12) to one of three canonical forms by well-known transformations of the independent variables (Mikhlin (1970)).

(i) $b^{2}-a c=0$ in $D$ (parabolic case).

$$
\frac{\partial^{2} u}{\partial x^{2}}+\alpha(x, y) \frac{\partial u}{\partial x}+\beta(x, y) \frac{\partial u}{\partial y}+\gamma(x, y) u=0
$$

where $\beta(x, y)$ is of fixed sign in $D$, say $\beta(x, y)>0$ in $D$.

(ii) $b^{2}-a c>0$ in $D$ (hyperbolic case).

$$
\frac{\partial^{2} u}{\partial x \partial y}+\alpha(x, y) \frac{\partial u}{\partial x}+\beta(x, y) \frac{\partial u}{\partial y}+\gamma(x, y) u=0 .
$$

(iii) $b^{2}-a c<0$ in $D$ (elliptic case).

$$
\frac{\partial^{2} u}{\partial x^{2}}+\frac{\partial^{2} u}{\partial y^{2}}+\alpha(x, y) \frac{\partial u}{\partial x}+\beta(x, y) \frac{\partial u}{\partial y}+\gamma(x, y) u=0 .
$$

We now study each of these three cases separately.

3. The parabolic equation. By known transformations of the dependent and independent variables any linear parabolic p.d.e. (13) can be reduced to an equivalent form

$$
\frac{\partial^{2} u^{\prime}}{\partial\left(x^{\prime}\right)^{2}}+\frac{\partial u^{\prime}}{\partial y^{\prime}}+G\left(x^{\prime}, y^{\prime}\right) u^{\prime}=0
$$

where

$$
\begin{aligned}
& x^{\prime}=\phi(x, y)=\int^{x}[\beta(z, y)]^{1 / 2} d z, \\
& y^{\prime}=y, \\
& u^{\prime}=e^{-C\left(x^{\prime}, y^{\prime}\right)} u, \\
& G\left(x^{\prime}, y^{\prime}\right)=\frac{\partial C}{\partial y^{\prime}}+\frac{\partial^{2} C}{\partial\left(x^{\prime}\right)^{2}}-\left(\frac{\partial C}{\partial x^{\prime}}\right)^{2}+\frac{\gamma(x, y)}{\beta(x, y)},
\end{aligned}
$$

and $C\left(x^{\prime}, y^{\prime}\right)$ is such that

$$
\frac{\partial C}{\partial x^{\prime}}=-\frac{1}{2}\left[\frac{\partial \phi}{\partial y}+\alpha(x, y)[\beta(x, y)]^{-1 / 2}+\frac{1}{2} \beta^{-3 / 2} \frac{\partial \beta}{\partial x}\right] .
$$

For convenience of notation we unprime the primed variables of p.d.e. (16) and consider

$$
\frac{\partial^{2} u}{\partial x^{2}}+\frac{\partial u}{\partial y}+G(x, y) u=0 .
$$

In Bluman (1980) it was shown explicitly that p.d.e. (17) is equivalent to the "backward" heat equation

$$
\frac{\partial^{2} U}{\partial \xi^{2}}+\frac{\partial U}{\partial \eta}=0
$$

if and only if $G(x, y)$ is of the form

$$
G(x, y)=q_{0}(y)+q_{1}(y) x+q_{2}(y) x^{2}
$$


where $\left\{q_{0}(y), q_{1}(y), q_{2}(y)\right\}$ are arbitrary functions of $y$, by mapping the infinitesimals of the group leaving invariant p.d.e. (17) into the infinitesimals of the 6-parameter group leaving invariant p.d.e. (18). We now show that the mapping algorithm described in $\S 2$ achieves this result in a much simpler way. Before showing this it should be noted that any constant coefficient parabolic p.d.e.

$$
\frac{\partial^{2} U^{\prime}}{\partial\left(\xi^{\prime}\right)^{2}}+2 A \frac{\partial U^{\prime}}{\partial \xi^{\prime}}+B \frac{\partial U^{\prime}}{\partial \eta^{\prime}}+C U^{\prime}=0
$$

is equivalent to (18) through the transformation

$$
U(\xi, \eta)=U^{\prime}\left(\xi^{\prime}, \eta^{\prime}\right) e^{\lambda \xi^{\prime}+\mu \eta^{\prime}}, \quad \xi=\xi^{\prime}, \quad \eta=\frac{\eta^{\prime}}{B}
$$

where $\lambda=A, \mu=\left(C-A^{2}\right) / B$. Thus the problem of mapping p.d.e. (13) into a constant coefficient p.d.e. reduces to using the algorithm of $\S 2$ to find all functions $G(x, y)$ and corresponding mappings (3) such that p.d.e. (17) is equivalent to p.d.e. (18).

Equation (17) is invariant under the Lie group of point transformations

$$
\begin{aligned}
& x^{*}=x+\varepsilon X(x, y)+O\left(\varepsilon^{2}\right), \\
& y^{*}=y+\varepsilon Y(x, y)+O\left(\varepsilon^{2}\right), \\
& u^{*}=u+\varepsilon g(x, y) u+O\left(\varepsilon^{2}\right)
\end{aligned}
$$

where the determining equations for the group infinitesimals $\{X, Y, g\}$ reduce to

$$
\begin{aligned}
& Y(x, y)=\tau(y), \\
& X(x, y)=\frac{1}{2} x \tau^{\prime}(y)+A(y), \\
& g(x, y)=\frac{x^{2} \tau^{\prime \prime}(y)}{8}+\frac{1}{2} x A^{\prime}(y)+B(y),
\end{aligned}
$$

and with respect to a given $G(x, y),\{\tau(y), A(y), B(y)\}$ satisfy

$$
\begin{aligned}
& \frac{x^{2} \tau^{\prime \prime \prime}(y)}{8}+\frac{1}{4} \tau^{\prime \prime}(y)+\frac{1}{2} x A^{\prime \prime}(y)+B^{\prime}(y) \\
& \quad+\left[\frac{1}{2} x \tau^{\prime}(y)+A(y)\right] \frac{\partial G}{\partial x}+\tau(y) \frac{\partial G}{\partial y}+\tau^{\prime}(y) G=0 .
\end{aligned}
$$

(In equations (23), (24) a prime denotes differentiation with respect to $y$.) In terms of the notation of $\S 2$,

$$
\begin{aligned}
& Y_{i}(x, y)=\tau_{i}(y), \\
& X_{i}(x, y)=\frac{1}{2} x \tau_{i}^{\prime}(y)+A_{i}(y), \\
& g_{i}(x, y)=\frac{x^{2} \tau_{i}^{\prime \prime}(y)}{8}+\frac{1}{2} x A_{i}^{\prime}(y)+B_{i}(y), \quad i=1,2 .
\end{aligned}
$$

The consistency relations $(11 \mathrm{~b}, \mathrm{a})$ are

$$
\begin{aligned}
& \tau_{1}(y) \tau_{2}^{\prime}(y)=\tau_{2}(y) \tau_{1}^{\prime}(y), \\
& A_{2}(y) \tau_{1}^{\prime}(y)+2 A_{1}^{\prime}(y) \tau_{2}(y)=A_{1}(y) \tau_{2}^{\prime}(y)+2 A_{2}^{\prime}(y) \tau_{1}(y),
\end{aligned}
$$


of which the general solution is

$$
\tau_{1}(y)=k \tau_{2}(y), \quad A_{1}(y)=k A_{2}(y)+l\left[\tau_{2}(y)\right]^{1 / 2}
$$

where $k, l$ are constants. Without loss of generality (w.l.o.g) we can set $k=0, l=1$ by an appropriate linear transformation of the variables $(\xi, \eta)$, namely $\bar{\xi}=l \xi, \bar{\eta}=\eta+k \xi$ and then unbarring the barred variables. Thus w.1.o.g.

$$
\tau_{1}(y)=0, \quad A_{1}(y)=\left[\tau_{2}(y)\right]^{1 / 2} .
$$

With respect to invariance under the infinitesimals corresponding to parameter $\varepsilon_{1}$, (24) becomes

$$
\frac{1}{2} x A_{1}^{\prime \prime}(y)+B_{1}^{\prime}(y)+A_{1}(y) \frac{\partial G}{\partial x}=0 .
$$

Hence it is necessary that

$$
\frac{\partial^{3} G}{\partial x^{3}} \equiv 0
$$

leading to $G(x, y)$ necessarily satisfying (19). Moreover (19) and (29) lead to $A_{1}(y)$ (hence $\left.\tau_{2}(y)\right)$ and $B_{1}(y)$ satisfying

$$
\begin{aligned}
& A_{1}^{\prime \prime}(y)+4 q_{2}(y) A_{1}(y)=0, \\
& B_{1}^{\prime}(y)=-q_{1}(y) A_{1}(y) .
\end{aligned}
$$

The other consistency relation $(11 \mathrm{c})$ leads to

$$
A_{2}^{\prime}(y) A_{1}(y)-A_{2}(y) A_{1}^{\prime}(y)=2 B_{1}^{\prime}(y) \tau_{2}(y) .
$$

Hence

$$
A_{2}(y)=\left[2 B_{1}(y)+m\right] A_{1}(y)
$$

for some constant $m$.

Finally, with respect to invariance under the infinitesimals corresponding to parameter $\varepsilon_{2}$, equation (24) leads to $B_{2}(y)$ satisfying

$$
B_{2}^{\prime}(y)=-\frac{1}{4} \tau_{2}^{\prime \prime}(y)-\left[\tau_{2}(y) q_{0}(y)\right]^{\prime}-A_{2}(y) q_{1}(y) .
$$

Moreover one sees that it is necessary and sufficient that $G(x, y)$ is of the form (19).

(a) Construction of the mapping. Equation (9d) leads to

$$
\frac{\partial \eta}{\partial x} \equiv 0 \Rightarrow \eta=\eta(y) \text {. }
$$

Equation (9c) then leads to

$$
\eta^{\prime}(y)=\frac{1}{\tau_{2}(y)} .
$$

From (28), (31) and (37) we see that $\eta(y)$ is a solution of the differential equation

$$
2 \eta^{\prime \prime \prime} \eta^{\prime}-3\left(\eta^{\prime \prime}\right)^{2}-16 q_{2}(y)\left(\eta^{\prime}\right)^{2}=0 \text {. }
$$

The solution of this differential equation is discussed in Bluman (1980).

Equations $(9 a, b)$ lead to

$$
\xi(x, y)=x\left[\eta^{\prime}(y)\right]^{1 / 2}+D(y)
$$


where

$$
D^{\prime}(y)=-A_{2}(y)\left[\eta^{\prime}(y)\right]^{3 / 2} .
$$

Finally, (7e, f) lead to the multiplier $F(\xi, \eta)$ satisfying

$$
\log F(\xi, \eta)=\frac{1}{4} x^{2} \frac{A_{1}^{\prime}(y)}{A_{1}(y)}+x \frac{B_{1}(y)}{A_{1}(y)}+W(y)
$$

where

$$
W^{\prime}(y)=B_{2}(y) \eta^{\prime}(y)-A_{2}(y) B_{1}(y)\left[\eta^{\prime}(y)\right]^{3 / 2} .
$$

It turns out that the corresponding $U(\xi, \eta)$ of (3) satisfies the constant coefficient p.d.e.

$$
\frac{\partial^{2} U}{\partial \xi^{2}}+\frac{\partial U}{\partial \eta}-m \frac{\partial U}{\partial \xi}+p U=0
$$

where the constant

$$
p=\frac{1}{2} A_{1}^{\prime}(y) A_{1}(y)-\left[B_{1}(y)\right]^{2}+B_{2}(y)-m B_{1}(y)+q_{0}(y)\left[A_{1}(y)\right]^{2} .
$$

A discussion of various aspects of this mapping and special cases is given in Bluman (1980).

4. The hyperbolic equation. Equation (14) is invariant under a Lie group of point transformations of the form (22) where for given coefficients $\{\alpha(x, y), \beta(x, y)$, $\gamma(x, y)\}$ the determining equations for the infinitesimals $\{X, Y, g\}$ reduce to

$$
\begin{aligned}
& X(x, y)=I(x), \quad Y(x, y)=J(y), \\
& \frac{\partial g}{\partial x}=-\beta I^{\prime}(x)-\frac{\partial \beta}{\partial x} I(x)-\frac{\partial \beta}{\partial y} J(y), \\
& \frac{\partial g}{\partial y}=-\alpha J^{\prime}(y)-\frac{\partial \alpha}{\partial y} J(y)-\frac{\partial \alpha}{\partial x} I(x), \\
& \frac{\partial^{2} g}{\partial x \partial y}+\alpha \frac{\partial g}{\partial x}+\beta \frac{\partial g}{\partial y}=0, \\
& \gamma\left[I^{\prime}(x)+J^{\prime}(y)\right]+\frac{\partial \gamma}{\partial x} I(x)+\frac{\partial \gamma}{\partial y} J(y)=0 .
\end{aligned}
$$

(A prime denotes differentiation with respect to the indicated variable.)

In terms of the notation of $\S 2$,

$$
X_{i}(x, y)=I_{i}(x), \quad Y_{i}(x, y)=J_{i}(y), \quad i=1,2 .
$$

The consistency relations $(11 \mathrm{a}, \mathrm{b})$ are

$$
\begin{gathered}
J_{1}^{\prime}(y) J_{2}(y)=J_{2}^{\prime}(y) J_{1}(y), \\
I_{1}^{\prime}(x) I_{2}(x)=I_{2}^{\prime}(x) I_{1}(x)
\end{gathered}
$$

of which the general solution is

$$
J_{2}(y)=k J_{1}(y), \quad I_{1}(x)=l I_{2}(x)
$$

where $k$ and $l$ are constants. Without loss of generality we can set $k=l=0$ corresponding to the linear transformation $\bar{\xi}=\xi+k \eta, \bar{\eta}=\eta+l \xi$ followed by unbarring the barred 
variables. Thus w.l.o.g.

$$
J_{2}(y) \equiv 0, \quad I_{1}(x) \equiv 0 .
$$

Hence from $(43 \mathrm{~b}, \mathrm{c})$ the corresponding $\left\{g_{1}(x, y), g_{2}(x, y)\right\}$ satisfy

$$
\begin{aligned}
& \frac{\partial g_{1}}{\partial x}=-\frac{\partial \beta}{\partial y} J_{1}(y), \\
& \frac{\partial g_{1}}{\partial y}=-\alpha J_{1}^{\prime}(y)-\frac{\partial \alpha}{\partial y} J_{1}(y), \\
& \frac{\partial g_{2}}{\partial x}=-\beta I_{2}^{\prime}(x)-\frac{\partial \beta}{\partial x} I_{2}(x), \\
& \frac{\partial g_{2}}{\partial y}=-\frac{\partial \alpha}{\partial x} I_{2}(x) .
\end{aligned}
$$

The consistency relation (11c) and equations (48a, d) lead to $\{\alpha(x, y), \beta(x, y)\}$, satisfying the first necessary condition

$$
\frac{\partial \beta}{\partial y}=\frac{\partial \alpha}{\partial x}
$$

Let

$$
\delta(x, y)=\frac{\partial \alpha}{\partial x}+\alpha \beta .
$$

Equation (43d) for $g=g_{1}, g_{2}$ leads to the following second necessary condition relating $\{\alpha(x, y), \beta(x, y)\}$ :

$$
\frac{\partial \delta}{\partial y} J_{1}(y)+\delta J_{1}^{\prime}(y)=0, \quad \frac{\partial \delta}{\partial x} I_{2}(x)+\delta I_{2}^{\prime}(x)=0 .
$$

Equations (51) are equivalent to $\delta(x, y)$ satisfying

$$
\frac{\partial^{2}}{\partial x \partial y}[\log \delta(x, y)]=0
$$

In particular, from (51),

$$
\delta(x, y)=\frac{m}{I_{2}(x) J_{1}(y)}
$$

for some constant $m$. Similarly from (43e) one sees that

$$
\gamma(x, y)=\frac{p}{I_{2}(x) J_{1}(y)}
$$

for some constant $p$.

(a) Construction of the mapping; basic theorem. Equations (9a, b, c, d) reduce to

$$
\begin{aligned}
& J_{1}(y) \frac{\partial \xi}{\partial y}=1, \\
& I_{2}(x) \frac{\partial \xi}{\partial x}=0,
\end{aligned}
$$




$$
\begin{aligned}
& I_{2}(x) \frac{\partial \eta}{\partial x}=1 \\
& J_{1}(y) \frac{\partial n}{\partial y}=0 .
\end{aligned}
$$

Hence the new variables are

$$
\begin{aligned}
& \xi(x, y)=\xi(y)=\int \frac{1}{J_{1}(y)} d y, \\
& \eta(x, y)=\eta(x)=\int \frac{1}{I_{2}(x)} d x .
\end{aligned}
$$

In the hyperbolic case the solution of $(7 \mathrm{e}, \mathrm{f})$ leads to the multiplier $F(\xi, \eta)$ satisfying the consistent pair of equations

$$
\begin{aligned}
& \frac{1}{F} \frac{\partial F}{\partial y}=-\alpha(x, y)+\frac{q}{J_{1}(y)}, \\
& \frac{1}{F} \frac{\partial F}{\partial x}=-\beta(x, y)+\frac{r}{I_{2}(x)}
\end{aligned}
$$

where $q, r$ are arbitrary constants.

The corresponding $U(\xi, \eta)$ of the mapping (3) satisfies the constant coefficient p.d.e.

$$
\frac{\partial^{2} U}{\partial \xi \partial \eta}+r \frac{\partial U}{\partial \xi}+q \frac{\partial U}{\partial \eta}+[q r+p-m] U=0
$$

It is convenient to choose $q=r=0$.

In summary we have the following basic theorem:

THEOREM. The hyperbolic p.d.e. (14) is equivalent to a constant coefficient p.d.e., in particular

$$
\frac{\partial^{2} U}{\partial \xi \partial \eta}+(p-m) U=0
$$

if and only if its coefficients $\{\alpha(x, y), \beta(x, y), \gamma(x, y)\}$ satisfy

$$
\frac{\partial \beta}{\partial y}=\frac{\partial \alpha}{\partial x}, \quad \frac{\partial \alpha}{\partial x}+\alpha \beta=m K^{\prime}(x) L^{\prime}(y), \quad \gamma(x, y)=p K^{\prime}(x) L^{\prime}(y)
$$

for some differentiable functions $\{K(x), L(y)\}$ and constants $m, p$. The mapping of the corresponding p.d.e. (14) to p.d.e. (59) is given by

$$
\xi=\xi(y)=L(y), \quad \eta=\eta(x)=K(x), \quad U(\xi, \eta)=u(x, y) H(x, y)
$$

where multiplier $H(x, y)$ satisfies

$$
\frac{1}{H} \frac{\partial H}{\partial y}=\alpha(x, y), \quad \frac{1}{H} \frac{\partial H}{\partial x}=\beta(x, y) .
$$

The first two equations of (60) are equivalent to $\alpha(x, y)$ satisfying

$$
\frac{\partial}{\partial y}\left[\frac{m K^{\prime}(x) L^{\prime}(y)-\partial \alpha / \partial x}{\alpha}\right]=\frac{\partial \alpha}{\partial x}
$$


with

$$
\beta(x, y)=\frac{m K^{\prime}(x) L^{\prime}(y)-\partial \alpha / \partial x}{\alpha} .
$$

Note that if $p=m=0$, then w.1.o.g. $L^{\prime}(y)=K^{\prime}(x) \equiv 1$, and hence $\xi=y, \eta=x$.

We now consider three particular cases:

(i) $p=m=0$; mapping to the wave equation. If $\gamma(x, y) \equiv 0$, the above theorem leads to showing that p.d.e. (13) can be mapped into the wave equation

$$
\frac{\partial^{2} U}{\partial \xi \partial \eta}=0
$$

if and only if

$$
\alpha(x, y)=\frac{B^{\prime}(y)}{A(x)+B(y)}, \quad \beta(x, y)=\frac{A^{\prime}(x)}{A(x)+B(y)}
$$

where $\{A(x), B(y)\}$ are arbitrary differentiable functions. The mapping is given by

$$
\xi=y, \quad \eta=x, \quad U(\xi, \eta)=u(x, y) H(x, y)
$$

where

$$
H(x, y)=A(x)+B(y) .
$$

(ii) $\alpha=\alpha(x)$, an arbitrary differentiable function of $x$. In this case, from (60), one can show that it is necessary and sufficient that

$$
\beta(x, y)=y \alpha^{\prime}(x)+h(x)
$$

where

$$
h(x)=c \alpha^{\prime}(x)-\frac{\alpha^{\prime}(x)}{\alpha(x)}
$$

for some constant $c$. Correspondingly

$$
K^{\prime}(x)=\alpha(x) \alpha^{\prime}(x), \quad L^{\prime}(y)=y+c, \quad m=1
$$

and

$$
\gamma(x, y)=p \alpha(x) \alpha^{\prime}(x)(y+c) .
$$

The mapping to (59) is given by

$$
\xi=\frac{y^{2}}{2}+c y, \quad \eta=\frac{\alpha^{2}(x)}{2}, \quad U(\xi, \eta)=u(x, y) H(x, y)
$$

where

$$
H(x, y)=\frac{e^{\alpha(x)(y+c)}}{\alpha(x)} .
$$

(iii) $\alpha=\alpha(y)$, an arbitrary differentiable function of $y$. In this case, from (60), it is necessary and sufficient that $\beta(x, y)=\beta(x)$, an arbitrary differentiable function of $x$. Correspondingly

$$
K^{\prime}(x)=\beta(x), \quad L^{\prime}(y)=\alpha(y), \quad m=1
$$


and

$$
\gamma(x, y)=p \beta(x) \alpha(y) .
$$

The mapping to (59) is given by

$$
\xi=\xi(y)=\int \alpha(y) d y, \eta=\eta(x)=\int \beta(x) d x, \quad U(\xi, \eta)=u(x, y) H(x, y)
$$

where

$$
H(x, y)=e^{[\xi(y)+\eta(x)]}
$$

(iv) An important corollary. The following corollary is an obvious consequence of the basic theorem of this section:

COROllary. The p.d.e.

$$
\frac{\partial^{2} u}{\partial x \partial y}+\gamma(x, y) u=0
$$

can be mapped into a constant coefficient p.d.e. by some transformation of the form (3) if and only if $\gamma(x, y)$ is separable in the form

$$
\gamma(x, y)=K^{\prime}(x) L^{\prime}(y)
$$

for some differentiable functions $\{K(x), L(y)\}$. The mapping is given by (61) with multiplier $H \equiv 1$.

(b) A particular class of hyperbolic equations. We apply the theorem of this section to the particular class of hyperbolic p.d.e's.

$$
C^{2}(x, t) \frac{\partial^{2} u}{\partial x^{2}}-\frac{\partial^{2} u}{\partial t^{2}}=0
$$

The criteria represented by (60) show that p.d.e. (73) can be mapped into a constant coefficient p.d.e. if and only if the following conditions (I) and (II) hold:

(I) The "wave speed" $C(x, t)$ satisfies

$$
\frac{\partial^{2} C}{\partial x \partial t}-\frac{1}{C} \frac{\partial C}{\partial x} \frac{\partial C}{\partial t}=0
$$

The general solution of p.d.e. (74) can be expressed in the form

$$
C(x, t)=\frac{g^{\prime}(t)}{f^{\prime}(x)}>0
$$

for arbitrary differentiable functions $\{g(t), f(x)\}$.

(II) For $C(x, t)$ of the form (75), let

$$
v=f(x)+g(t), \quad w=f(x)-g(t) .
$$

$\{g(t), C(x, t)\}$ satisfy

$$
2 C \frac{\partial^{2} C}{\partial x^{2}}+\frac{2}{C} \frac{\partial^{2} C}{\partial t^{2}}-3\left(\frac{1}{C} \frac{\partial C}{\partial t}\right)^{2}-\left(\frac{\partial C}{\partial x}\right)^{2}=-16 m\left[g^{\prime}(t)\right]^{2} K^{\prime}(v) L^{\prime}(w)
$$

for some differentiable functions $\{K(v), L(w)\}$, constant $m$. 
If $C(x, t)$ satisfies $(75),(76),(77)$ then the transformation

$$
\begin{aligned}
& \xi(x, t)=\xi(w)=L(w), \\
& \eta(x, t)=\eta(v)=K(v), \\
& U(\xi, \eta)=u(x, t) H(x, t),
\end{aligned}
$$

where the multiplier

$$
H(x, t)=\left[f^{\prime}(x) g^{\prime}(t)\right]^{1 / 2}
$$

maps (70) into the constant coefficient p.d.e.

$$
\frac{\partial^{2} U}{\partial \xi \partial \eta}-m U=0 .
$$

(i) The case $m=0$, mapping to the wave equation. If $m=0$, one can show that p.d.e. (73) can be mapped into the wave equation (64) if and only if $C(x, t)$ is of the form

$$
C(x, t)=\frac{a_{0}+2 a_{1} x+a_{2} x^{2}}{b_{0}+2 b_{1} t+b_{2} t^{2}}
$$

where constants $\left\{a_{0}, a_{1}, a_{2}, b_{0}, b_{1}, b_{2}\right\}$ are related by

$$
\left(a_{1}\right)^{2}-a_{2} a_{0}=\left(b_{1}\right)^{2}-b_{2} b_{0}=\Delta \text {, say. }
$$

We examine separately the cases $\Delta=0, \Delta>0, \Delta<0$.

(I) $\Delta=0$. Here the mapping is given by

$$
\xi=\frac{1}{a_{2} x+a_{1}}-\frac{1}{b_{2} t+b_{1}}, \quad \eta=\frac{1}{a_{2} x+a_{1}}+\frac{1}{b_{2} t+b_{1}}, \quad U(\xi, \eta)=u(x, t) H(x, t)
$$

where the multiplier

$$
H(x, t)=\left[\left(a_{2} x+a_{1}\right)\left(b_{2} t+b_{1}\right)\right]^{-1 / 2} .
$$

(II) $\Delta=\rho^{2}>0$. Here the mapping is given by

$$
\begin{aligned}
& \xi=\log \left|\frac{a_{2} x+a_{1}-\rho}{a_{2} x+a_{1}+\rho}\right|^{\rho / 2}-\log \left|\frac{b_{2} t+b_{1}-\rho}{b_{2} t+b_{1}+\rho}\right|^{\rho / 2}, \\
& \eta=\log \left|\frac{a_{2} x+a_{1}-\rho}{a_{2} x+a_{1}+\rho}\right|^{\rho / 2}+\log \left|\frac{b_{2} t+b_{1}-\rho}{b_{2} t+b_{1}+\rho}\right|^{\rho / 2}, \\
& U(\xi, \eta)=u(x, t) H(x, t)
\end{aligned}
$$

where

$$
H(x, t)=\left[\left(a_{1}+2 a_{1} x+a_{2} x^{2}\right)\left(b_{0}+2 b_{1} t+b_{2} t^{2}\right)\right]^{-1 / 2} .
$$

(III) $\Delta=-\rho^{2}<0$. Here the mapping to the wave equation is given by

$$
\begin{aligned}
& \xi=\frac{1}{a_{2} \rho} \arctan \left(\frac{a_{2} x+a_{1}}{a_{2} \rho}\right)-\frac{1}{b_{2} \rho} \arctan \left(\frac{b_{2} t+b_{1}}{b_{2} \rho}\right), \\
& \eta=\frac{1}{a_{2} \rho} \arctan \left(\frac{a_{2} x+a_{1}}{a_{2} \rho}\right)+\frac{1}{b_{2} \rho} \arctan \left(\frac{b_{2} t+b_{1}}{b_{2} \rho}\right), \\
& U(\xi, \eta)=u(x, t) H(x, t)
\end{aligned}
$$


where the multiplier

$$
H(x, t)=\left[\left(a_{0}+2 a_{1} x+a_{2} x^{2}\right)\left(b_{0}+2 b_{1} t+b_{2} t^{2}\right)\right]^{-1 / 2} .
$$

(ii) The special case $C(x, t)=C(x)$. In this case one can show that

$$
C^{2}(x) \frac{\partial^{2} u}{\partial x^{2}}-\frac{\partial^{2} u}{\partial t^{2}}=0
$$

maps into a constant coefficient p.d.e. if and only if $C(x)$ satisfies the differential equation

$$
\frac{d}{d x}\left[\frac{C^{2} C^{\prime \prime \prime}}{2 C C^{\prime \prime}-\left(C^{\prime}\right)^{2}}\right]=0
$$

Let $c(x)$ be such that $c^{\prime}(x)=1 / C(x)$. Then equation (86) is equivalent to

$$
2 C C^{\prime \prime}-\left(C^{\prime}\right)^{2}=-16 m e^{2 a c(x)}
$$

for some constants $a, m$. The transformation

$$
\begin{aligned}
& \xi(x, t)=\frac{e^{a[c(x)-t]}-a}{a}, \\
& \eta(x, t)=\frac{e^{a[c(x)+t]}-a}{a}, \\
& U(\xi, \eta)=[C(x)]^{-1 / 2} u(x, t)
\end{aligned}
$$

maps p.d.e. (85) into p.d.e. (80).

5. The elliptic equation. In the case of the elliptic p.d.e. (15), the use of complex variables $z=x+i y, \bar{z}=x-i y$, reduces the computations to those for the hyperbolic equation (14). In particular the corresponding basic theorem is as follows:

THEOREM. The elliptic p.d.e. (15) is equivalent to a constant coefficient p.d.e. if and only if its coefficients $\{\alpha(x, y), \beta(x, y), \gamma(x, y)\}$ satisfy

$$
\begin{aligned}
& \frac{\partial \beta}{\partial x}=\frac{\partial \alpha}{\partial y}, \\
& 2\left(\frac{\partial \alpha}{\partial x}+\frac{\partial \beta}{\partial y}\right)+\alpha^{2}+\beta^{2}=4 m\left|K^{\prime}(z)\right|^{2}, \\
& \gamma(x, y)=p\left|K^{\prime}(z)\right|^{2}
\end{aligned}
$$

for some analytic function $K(z)$ and real constants $m, p$. If the coefficients of the elliptic p.d.e. (15) satisfy (89), the transformation

$$
\xi+i \eta=K(z), \quad U(\xi, \eta)=u(x, y) H(x, y),
$$

where the multiplier $H(x, y)$ satisfies

$$
\frac{2}{H} \frac{\partial H}{\partial x}=\alpha(x, y), \quad \frac{2}{H} \frac{\partial H}{\partial y}=\beta(x, y),
$$

maps equation (15) into the Helmholtz equation

$$
\frac{\partial^{2} U}{\partial \xi^{2}}+\frac{\partial^{2} U}{\partial \eta^{2}}+(p-m) U=0 .
$$

Note that if $p=m=0$, one can have $\xi=x, \eta=y$. 
(a) An interesting special case. If $\alpha(x, y)$ and $\beta(x, y)$ are harmonic conjugates, in particular

$$
\beta+i \alpha=2 \lambda K^{\prime}(z) \text { and } \gamma=p\left|K^{\prime}(z)\right|^{2}
$$

for some constants $\lambda, p$, then conditions (89) are satisfied and the transformation

$$
\xi+i \eta=K(z), \quad U(\xi, \eta)=u(x, y) e^{\lambda \eta}
$$

maps p.d.e. (15) into the Helmholtz equation

$$
\frac{\partial^{2} U}{\partial \xi^{2}}+\frac{\partial^{2} U}{\partial \eta^{2}}+\left(p-\lambda^{2}\right) U=0
$$

Acknowledgment. The author is grateful for support from the Science and Engineering Research Council of the U.K. and NSERC of Canada and is most grateful for the warm hospitality of the staff of the Department of Theoretical Mechanics at the University of Nottingham during his sabbatical visit in 1981-82.

\section{REFERENCES}

G. W. BLUMAN (1980) On the transformation of diffusion processes into the Wiener process, this Journal, 39, pp. 238-247.

G. W. Bluman AND J. D. COLE (1974), Similarity Methods for Differential Equations, Springer-Verlag, New York, Heidelberg, Berlin.

S. G. Mikhlin (1970), Matkematical Physics, an Advanced Course, North-Holland, Amsterdam, London.

L. V. OvsJannikov (1962) Group Properties of Differential Equations, Siberian Branch of the U.S.S.R. Academy of Sciences, Novosibirsk. (In Russian, translated by G. W. Bluman.) 